\title{
INFECÇÃO POR COVID-19 DURANTE A GESTAÇÃO: AVALIAÇÃO DAS MANIFESTAÇÕES CLÍNICAS E DESFECHO GESTACIONAL
}

\author{
* Maria Eduarda Serafim Crispim ' \\ Alice Cabral Frade ${ }^{\text {I }}$ \\ Camila Araújo de Vasconcellos ${ }^{\prime}$ \\ José Raimundo Ferreira Neto I \\ Marina Brandão Ramalho de Brito"
}

\section{RESUMO}

A pandemia do novo coronavírus (SARS-CoV-2) e sua doença respiratória (COVID-19) logo tornaram-se uma ameaça global. Por tratar-se de uma nova doença, a busca por evidências que indiquem a avaliação e o manejo adequados, principalmente para os grupos de risco, torna-se imprescindível. Portanto, este trabalho objetiva elucidar e analisar os conhecimentos atuais sobre o impacto da contaminação pelo vírus durante a gestação para seu devido enfrentamento. Realizou-se uma revisão sistemática que teve como princípio norteador a análise das manifestações clínicas e complicações ocorridas nas gestantes infectadas por SARS-CoV-2. Para tal, foram utilizados os descritores: "Pregnancy" AND "Coronavirus", através das bases MEDLINE e SciELO. A partir dos critérios de inclusão e exclusão foram selecionadas 15 produções. As manifestações mais comuns foram similares às das mulheres não-gestantes: febre, fadiga, dispneia, mialgia e tosse. Entre divergências, notou-se a ocorrência de agravamentos, como a pré-eclâmpsia. Apesar dessas contradições na literatura e limitações quantitativas das amostras, as complicações gestacionais mais relatadas foram sofrimento fetal, ruptura prematura de membranas, partos prematuros e até mesmo morte fetal. Todavia, achados de transmissão vertical não foram observados. Apesar de significativo aumento na realização de cesáreas, as escolhas devem ser individualizadas, uma vez que a infecção por COVID-19 não é isoladamente indicativo de intervenção cirúrgica. Pontua-se que a maioria dos estudos são provenientes de regiões bem distintas cultural e demograficamente, mas o conhecimento e análise da literatura são essenciais para entendimento da doença e delineamento das condutas adequadas.

PALAVRAS-CHAVE: Infecções por Coronavírus. Complicações Infecciosas na Gravidez. Manifestações Clínicas.

Graduandos. Faculdade de Medicina Nova Esperança Esperança, FAMENE. Departamento de MEDICINA. CEP: 58067-698, João Pessoa, Paraíba, Brasil. *Autor correspondente: me_serafimcrispim@hotmail.com. ORCID ID: 0000-0001-5647-748X; 0000-0001-5454-8512; 0000-0001-6192-8652; 0000-0003-0687-1870.

Médica graduada pela Faculdade de Medicina Nova Esperança, FAMENE. CEP: 58067-698, João Pessoa, Paraíba, Brasil. Orcid: 0000-0001-7831-821X. 


\section{INTRODUÇÃO}

Em março de 2020, a Organização Mundial de Saúde (OMS) ${ }^{1}$ declarou pandemia da COVID-19, doença respiratória causada pelo novo coronavírus SARS-CoV-2,2,3,4 que já se constituía em epidemia na Ásia e espalhou-se pelo mundo. $O$ primeiro caso foi identificado na cidade de Wuhan, China, em dezembro de $2019^{3}$ e, em decorrência do alto poder de transmissibilidade do vírus, em meados de abril do mesmo ano, a doença já havia sido registrada nos cinco continentes. 5

Por esse motivo, líderes de todo o mundo elaboraram estratégias para controlar a disseminação da doença, tais como distanciamento social obrigatório, abertura apenas de serviços essenciais, e até mesmo atendimento médico apenas para casos urgentes. Entretanto, apesar dos esforços, a disseminação da COVID-19 continua em andamento. 5,6

As manifestações clínicas da COVID-19 variam desde casos assintomáticos, a infecções leves das vias aéreas superiores, a até casos graves cursando com pneumonia e insuficiência respiratória aguda. 5 podendo levar a óbito. ${ }^{6}$ Indivíduos que apresentam doenças e/ou comorbidades prévias, idosos e imunodeficientes possuem maior risco desses

\section{MATERIAL E MÉTODOS}

\section{Seleção dos estudos}

Esta é uma revisão sistemática baseada no método PRISMA 9 que teve como objetivo revisar a literatura obtida, acerca de coronavírus e gravidez, até 15 de Outubro de 2020. O estudo teve como questão norteadora: Quais são as manifestações cursos mais graves ou fatais da COVID-19.5,6

Com base em surtos anteriores do coronavírus, comosíndromerespiratóriaaguda grave pelo SARS-CoV, que ocorreu em 2003, e a síndrome respiratória do Oriente Médio, devido ao MERS-CoV em 2012, acredita-se que mulheres grávidas, puérperas e neonatos são populações consideradas vulneráveis e, dessa maneira, mais suscetíveis a desenvolver a forma grave do novo coronavírus (SARSCoV-2). 3,4,7,8 Em relação as gestantes, essa vulnerabilidade está associada as alterações fisiológicas e a supressão imunológica inerentes a gestação que tornam as mães mais propensas a desenvolver diversas infecções com risco de complicação. 2,3,5,7 Além disso, alterações anatômicas como aumento do diâmetro transverso da caixa torácica e nível elevado do diafragma tornam a gestante intolerante a hipóxia. ${ }^{7}$

Por tratar-se de uma doença nova, com dados ainda escassos na literatura, torna-se relevante a busca por evidências que auxiliem no manejo e avaliação clínica das pacientes. Portanto, o objetivo deste artigo é elucidar e avaliar estudos atuais sobre as manifestações e complicações da COVID-19 em gestantes.

clínicas e complicações ocorridas nas gestantes infectadas por SARS-CoV-2? Para a seleção dos artigos, foram utilizados os descritores e o termo Medical Subject Heandig (MeSH): "Pregnancy" AND "Coronavirus" através das bases de dados eletrônicas MEDLINE e SciELO.

A partir dos descritores, foram identificados 989 artigos na base MEDLINE e 
outros oito artigos em outras bases de dados.

Após a eliminação dos duplicados, restaram 994 (100\%) artigos, dos quais 952 (95,7\%) foram excluídos por fugirem do tema. Dessa forma, $42(4,1 \%)$ estudos foram lidos integralmente, dos quais 15 (1,5\%) descumpriram os critérios de elegibilidade. Assim, 27 (2,6\%) trabalhos foram qualitativamente incluídos e 15 (1,5\%) artigos quantitativamente incluídos (revisão sistemática). O fluxograma visto na Figura 1 representa as fases de seleção dos estudos diante os critérios de elegibilidade.

\section{Critérios de seleção}

Para a elaboração desse artigo foram incluídos: ensaios clínicos randomizados, revisões sistemáticas e meta-análises. Os 15 artigos escolhidos foram escritos em inglês e publicados no ano de 2020. Posteriormente, foram adicionados trabalhos seguindo a técnica de snowballing. Foram excluídos artigos duplicados, relatos de casos, artigos envolvendo animais e que fugiam ao tema.

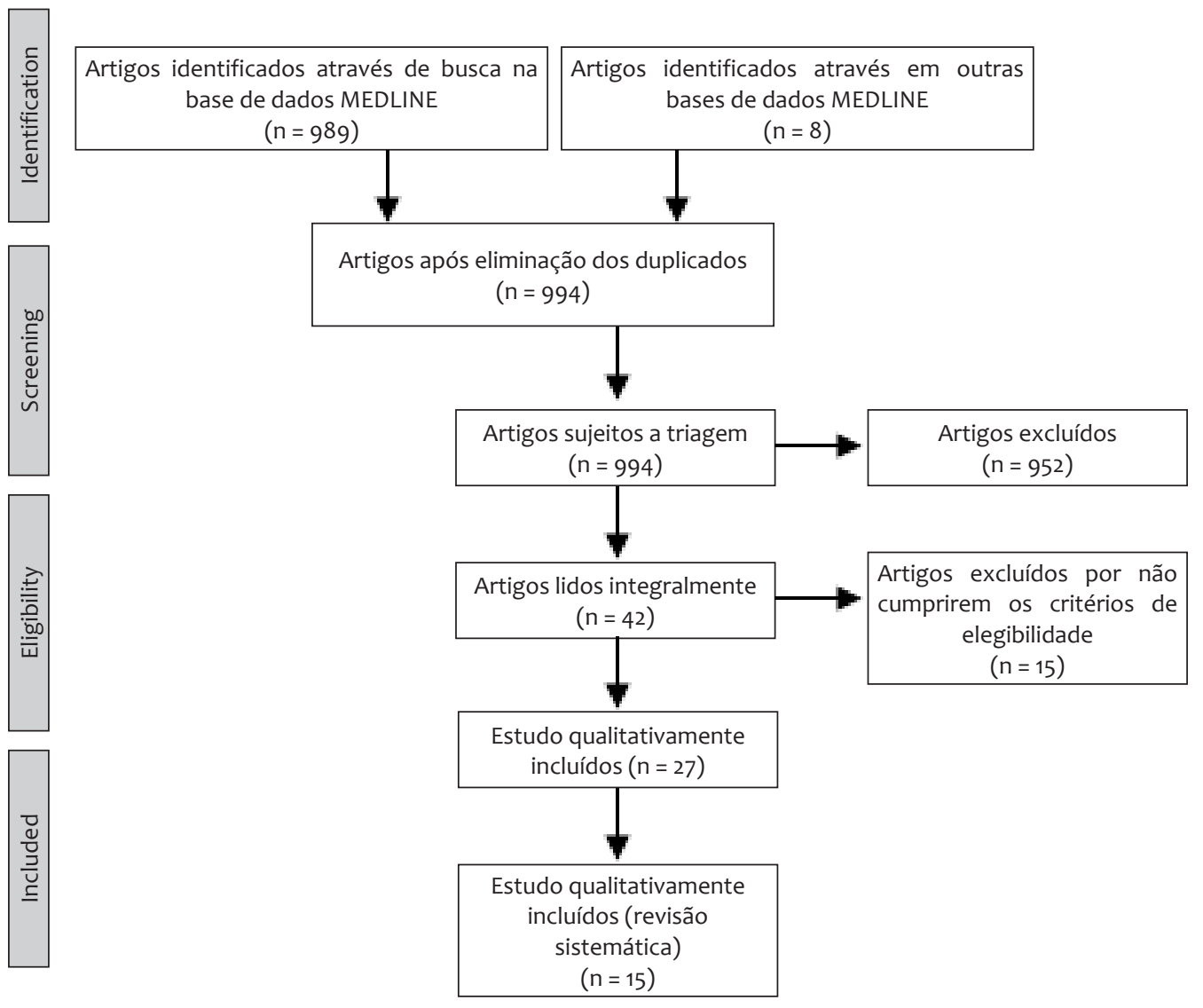

Figura 1: Fluxograma baseado no método PRISMA ${ }^{8}$ representando a seleção dos estudos incluídos na revisão sistemática 


\section{RESULTADOS E DISCUSSÃO}

A partir dos critérios de inclusão, exclusão e análise crítica foram selecionadas 15 produções científicas, categorizadas na Tabela 1, que contribuíram com o objeto do estudo. Os artigos encontrados possuem diversas nacionalidades, sendo elas: Itália
(3), Qatar (1), Estados Unidos (1), China (4), Etiópia (1), Irã (1), Austrália (1), Portugal (1), Suécia (1), Irlanda (1). Todos os estudos (100\%) foram publicados em inglês, lidos na íntegra e devidamente referenciados.

TABELA 1: Disposição dos artigos selecionados para compor a revisão integrativa, publicados no ano de 2020, considerando título, autores, país e banco de dados.

\begin{tabular}{|c|c|c|}
\hline TíTULO & AUTORES & TIPO DE ESTUDO \\
\hline $\begin{array}{l}\text { Maternal and neonatal outcomes associated with COVID-19 infection: } \\
\qquad \text { A systematic review }\end{array}$ & Vinayak Smith et al. ${ }^{2}$ & $\begin{array}{l}\text { Revisão sistemática } \\
\text { da literatura }\end{array}$ \\
\hline $\begin{array}{l}\text { Maternal and perinatal outcomes and pharmacological management } \\
\text { of Covid-19 infection in pregnancy: a systematic review protocol }\end{array}$ & Binny Thomas et al. ${ }^{3}$ & $\begin{array}{l}\text { Revisão sistemática } \\
\text { da literaturas }\end{array}$ \\
\hline $\begin{array}{c}\text { Coronavirus disease } 2019 \text { during pregnancy: a systematic review of } \\
\text { reported cases }\end{array}$ & $\begin{array}{l}\text { Anna Nunzia Della Gatta } \\
\text { et al. }{ }^{4}\end{array}$ & $\begin{array}{l}\text { Revisão sistemática } \\
\text { da literatura }\end{array}$ \\
\hline $\begin{array}{c}\text { Effects of COVID-19 Infection during Pregnancy and Neonatal } \\
\text { Prognosis: What Is the Evidence? }\end{array}$ & $\begin{array}{c}\text { Àlvaro Francisco Lopes } \\
\text { de Sousa et al. }\end{array}$ & $\begin{array}{l}\text { Revisão sistemática da } \\
\text { literatura }\end{array}$ \\
\hline $\begin{array}{l}\text { Prior and novel coronaviruses, Coronavirus Disease } 2019 \text { (COVID-19), } \\
\text { and human reproduction: what is known? }\end{array}$ & James Segars et al. ${ }^{6}$ & $\begin{array}{l}\text { Revisão sistemática da } \\
\text { literatura e meta-análise }\end{array}$ \\
\hline $\begin{array}{l}\text { Maternal and perinatal outcomes with COVID-19: A systematic review } \\
\text { of } 108 \text { pregnancies }\end{array}$ & Mehreen Zaigham et al. ${ }^{7}$ & $\begin{array}{l}\text { Revisão sistemática } \\
\text { da literatura }\end{array}$ \\
\hline $\begin{array}{l}\text { The efect of coronavirus infection (SARS-CoV-2, MERS-CoV, and SARS- } \\
\text { CoV) during pregnancy and the possibility of vertical }\end{array}$ & Kuma Diriba et al. ${ }^{8}$ & $\begin{array}{l}\text { Revisão sistemática da } \\
\text { literatura e meta-análise }\end{array}$ \\
\hline $\begin{array}{l}\text { Novel } 2019 \text { coronavírus SARS-COV-2 (COVID-19): An update overview } \\
\text { for emergency clinicians }\end{array}$ & AL Giwa et al. ${ }^{10}$ & $\begin{array}{l}\text { Revisão sistemática } \\
\text { da literatura }\end{array}$ \\
\hline $\begin{array}{l}\text { Effect of coronavirus disease } 2019 \text { (COVID-19) on maternal, perinatal } \\
\text { and neonatal outcome: systematic review }\end{array}$ & $J$ Juan et al. ${ }^{11}$ & $\begin{array}{l}\text { Revisão sistemática } \\
\text { da literatura }\end{array}$ \\
\hline $\begin{array}{l}\text { Clinical manifestations and outcome of SARS-CoV-2 infection during } \\
\text { pregnancy. }\end{array}$ & Yangli Liu et al. ${ }^{12}$ & Série de casos \\
\hline $\begin{array}{l}\text { Outcome of coronavirus spectrum infections (SARS, MERS, COVID-19) } \\
\text { during pregnancy: a systematic review and meta-analysis }\end{array}$ & Daniele Di Mascio et al. ${ }^{13}$ & $\begin{array}{l}\text { Revisão sistemática } \\
\text { da literatura }\end{array}$ \\
\hline A systematic review and meta-analysis & $\begin{array}{c}\text { Giampiero Capobianco } \\
\text { et al. }^{14}\end{array}$ & $\begin{array}{l}\text { Revisão sistemática da } \\
\text { literatura e meta-análise }\end{array}$ \\
\hline $\begin{array}{c}\text { Coronavirus Disease } 2019 \text { (COVID-19): A Systematic Review of } \\
\text { Pregnancy and the Possibility of Vertical Transmission }\end{array}$ & $\begin{array}{l}\text { Mohammad Ali Ashraf } \\
\text { et al. }{ }^{15}\end{array}$ & $\begin{array}{l}\text { Revisão sistemática } \\
\text { da literatura }\end{array}$ \\
\hline $\begin{array}{l}\text { Clinical characteristics and intrauterine vertical transmission potential } \\
\text { of COVID-19 infection in nine pregnant women: a retropective review } \\
\text { of medical records }\end{array}$ & Huijun Chen et al. ${ }^{16}$ & Série de casos \\
\hline $\begin{array}{c}\text { Clinical analysis of } 10 \text { neonates born to mothers with 2019-nCov } \\
\text { pneumonia }\end{array}$ & Huaping Zhu et al. ${ }^{17}$ & Série de casos \\
\hline
\end{tabular}

Com o advento da pandemia, tornou-se necessário investigar os possíveis efeitos da infecção por COVID-19, durante a gestação, principalmente no primeiro e segundo trimestres. Tanto as altas taxas de gestantes assintomáticas como a escassez 
de recomendações para a realização de testes de rotina ou rastreio de COVID-19 representam um desafio. Associado a isso, o pânico e o estresse causado pelo início da pandemia e o confinamento prolongado devem ser considerados na avaliação de todas as gestantes, infectadas ou não. Devido a esse cenário de incertezas, todos os cidadãos, incluindo as gestantes, devem seguir estritamente as recomendações das diretrizes. $O$ que se sabe, até então, é que tem relevância clínica e social classificar aqueles que precisam de teste diagnóstico, casos prováveis, suspeitos ou confirmados. ${ }^{10}$

Juan et al. ${ }^{11}$ discorreram sobre os sintomas mais frequentes em gestantes infectadas por COVID-19, sendo esses: febre, tosse, dispneia, fadiga e mialgia. Além disso, na admissão hospitalar, a maioria dos casos apresentou sombra irregular ou opacidade em vidro fosco à tomografia computadorizada (TC) de tórax, enquanto a contagem de leucócitos era normal ou baixa. Linfocitopenia e proteína $C$ reativa ( $P C R$ ) elevada foram os achados laboratoriais mais comuns. Também foi citado que a COVID-19 não se relaciona com o risco de resultados adversos na gravidez, como pré-eclâmpsia.

Corroborando com as manifestações clínicas encontradas no estudo de Juan et al., ${ }^{11}$ Liu et al. ${ }^{12}$ promoveram um estudo com 13 gestantes infectadas por SARS-CoV-2 entre dezembro de 2019 e fevereiro de 2020 na China. Dez pacientes (77\%) apresentaram febre que variava entre $37.3-39.0{ }^{\circ} \mathrm{C}$ acompanhada de fadiga, três (23\%) manifestaram dispneia e uma $(7,6 \%)$ desenvolveu pneumonia severa com disfunção severa de múltiplos órgãos necessitando de internação em UTI. Algumas gestantes receberam alta após o tratamento com a gravidez ainda em curso $(n=3)$, enquanto as outras pacientes $(n=10)$ foram submetidas a cesariana. Cinco (50\%) apresentaram complicações como: sofrimento fetal $(n=3)$, ruptura prematura de membranas $(n=1)$ e um natimorto $(n=1)$. Não foi observada asfixia neonatal grave ou indícios de transmissão vertical. $^{12}$

Já a revisão sistemática por Di Mascio et al., ${ }^{13}$ feita dentre as mães hospitalizadas infectadas por coronavírus, incluindo COVID-19, refere que o nascimento prematuro é o resultado adverso mais comum na gravidez, ocorrendo em $41,1 \%$ dos casos. A infecção também se relacionou com maior taxa de pré-eclâmpsia, cesárea e morte perinatal, o que contraria o estudo de Juan et al. ${ }^{11}$ Outro estudo, uma revisão sistemática e metaanálise feita por Capobianco et al., ${ }^{14}$ mostrou um alto risco de complicações maternas.

O número total de indivíduos recrutados na análise foi de 114 , que eram COVID-19 positivos. Os sintomas e sinais clínicos mais prevalentes foram: tosse, febre, mialgia, fadiga, dor de garganta, dispneia e linfopenia. A temperatura corporal em gestantes variou de 36,5 a $39,0{ }^{\circ} \mathrm{C}$. Embora tenham sido tratadas de forma heterogênea (de antibióticos, antivirais e corticosteroides, em diferentes combinações), 45,0\% das mulheres infectadas apresentaram complicações relacionadas a gravidez, sendo mais frequentes: testes fetais alterados, ruptura prematura de membranas, seguidos por placenta prévia e pré-eclâmpsia. Com esse trabalho, foi ratificado a relação do COVID-19 com complicações gestacionais, já citadas por Di Mascio et al. ${ }^{13}$

Ademais, Asharaf et al., 15 em revisão sistemática que incluiu 21 artigos com as características clínicas de 90 pacientes grávidas, indicaram que os sintomas mais comuns incluem febre, tosse e dispneia, assim como nos estudos de Juan et al. ${ }^{11} \mathrm{e}$ Liu et al. ${ }^{12}$ Ainda, corroborando os achados de Capobianco et al. ${ }^{14}$ e de Di Mascio et al. ${ }^{13}$ em relação ao maior risco do nascimento pré-termo e demais complicações materno- 
fetais, Asharaf et al. ${ }^{15}$ relacionam a COVID-19 às complicações gestacionais, sendo as mais comumente encontradas trabalho de parto prematuro e sofrimento fetal. Também indicaram que a cesárea foi realizada em mais da metade dos casos, mas não foi indicado o motivo da intervenção cirúrgica, de modo que se suspeita a necessidade real do procedimento. Também foi encontrado que uma proporção relativamente alta de mulheres grávidas (13,0\%) foi admitida na unidade de tratamento intensivo (UTI), todavia nenhum caso de morte foi notificado, o que pode ser explicado pelo fato de as pacientes infectadas serem jovens.

A revisão sistemática de Della Gatta et al. 4 também cita que o número de cesárias eletivas aumentou de forma provavelmente desnecessária. A maioria das pacientes, principalmente as mais jovens, são assintomáticas, ou não apresentam comprometimento respiratório. Autoridades e sociedades profissionais, como o Conselho Italiano de Saúde, o Royal College of Obstetricians e Ginecologistas Inglês, e a Society for Maternal-Fetal Medicine, assumiram que COVID-19 não é uma contraindicação ao parto vaginal. No entanto, praticamente todas as pacientes foram submetidas a parto cesáreo eletivo, após o diagnóstico. Em uma minoria de pacientes, o comprometimento respiratório grave estará presente. Nesses casos, após a viabilidade fetal, o parto cesáreo pode salvar a vida da mãe e do recém-nascido.

A revisão retrospectiva promovida por Chen et al. ${ }^{16}$ reuniu prontuários médicos de nove gestantes diagnosticadas com COVID-19, internadas no Hospital Zhongnan de 20 a 31 de Janeiro de 2020. As nove gestantes estavam no terceiro trimestre quando foram admitidas no hospital e apresentaram clínica similar aos casos documentados de adultas não-grávidas acometidas pelo vírus. Apesar da limitação quantitativa da amostra, não foram encontradas evidências de infecção intrauterina por transmissão vertical naquelas gestantes que foram infectadas no final da gravidez. Todas as nove gestantes foram submetidas à cesariana, dentre outras indicações, pelo risco incerto de transmissão materno-fetal durante o parto vaginal. Foi registrado o nascimento dos nove nascidos vivos, sem evidências de asfixia neonatal e todos obtiveram o score Apgar entre 8-9 no 10 minuto e entre 9-10 no 50 minuto de vida.

Já o estudo promovido por Zhu et al. ${ }^{17}$ analisou o desfecho de 10 neonatos nascidos de mães com infecção confirmada por COVID-19 em 5 diferentes hospitais e teve como resultado que a infecção pode estar relacionada com efeitos adversos nos recémnascidos. Foram identificados problemas como: parto prematuro $(n=6)$, dificuldade respiratória $(n=6)$, trombocitopenia associada a função hepática anormal $(n=2)$ e até morte $(n=1)$. Contudo, assim como no estudo de Chen et al., ${ }^{16}$ casos de transmissão vertical também não foram detectados. ${ }^{17}$

Lopes de Sousa Áf et al. 5 afirmam que das 755 gestantes e 598 neonatos nascidos durante o estudo, 379 (65\%) realizaram cesáreas. Além disso, a pré-eclâmpsia foi um achado em $19.1 \%$ dos casos, mas sem ruptura prematura das membranas ou restrição do crescimento fetal. Partos prematuros e morte perinatal corresponderam a $32,1 \%$ e $33,2 \%$, respectivamente. Ainda, não houve relatos de estresse fetal, Apgar $<7$ no 50 minuto, asfixia neonatal ou admissão na unidade de tratamento intensivo (UTI) neonatal. De 493 dos neonatos testados para o SARS-CoV-2, apenas 9 (2\%) tiveram resultados positivos. Contudo, não há evidência de transmissão vertical que possa ser corroborada, assim como nos estudos de Chen et al. ${ }^{16}$ e Zhu et al. ${ }^{17}$ 


\section{CONSIDERAÇÕES FINAIS}

A gestação gera diversas alterações fisiológicas na mulher, sendo uma delas a supressão imunológica que a torna mais suscetível a diversas infecções e com risco maior de complicação. Portanto, mães e recémnascidos requerem uma atenção especial na prevenção, diagnóstico e tratamento da COVID-19.

As manifestações clínicas mais comuns nas gestantes analisadas foram similares às das pacientes não-grávidas, sendo as principais: febre, fadiga, dispneia, mialgia e tosse. Há poucos relatos na literatura de pacientes que desenvolveram complicações pulmonares como pneumonia grave, porém a população estudada na maioria dos artigos era restrita.

Apesar das contradições entre literaturas e da limitação quantitativa da amostra estudada, algumas complicações gestacionais parecem estar associadas a infecção materna pelo novo coronavírus como: pré-eclâmpsia, sofrimento fetal, ruptura prematura de membranas, partos prematuros e até mesmo morte fetal o que torna necessário fornecer monitoramento fetal contínuo durante o trabalho de parto nesses casos. Já evidências de que transmissão vertical não foi observada nos estudos analisados.

A infecção por SARS-COV-2 em si não é uma indicação de parto cesáreo. A escolha do momento e da via de parto deve ser individualizada com base na gravidade da doença, condições fetais, comorbidades pré-existentes e história obstétrica. Contudo, vem se observando um número crescente de cesáreas no grupo de mulheres infectadas, mesmo sem indicação registrada.

Embora a quantidade de artigos e dados seja reduzida e, muitas vezes, provenientes de regiões nas quais a situação social e a demográfica é diferente no Brasil, o tema é de extrema importância considerando a atual conjuntura da pandemia. Ressaltase a necessidade de mais artigos com maior amostra populacional de estudo para dar maior confiabilidade aos achados referentes as manifestações clínicas e o desfecho gestacional das mães contaminadas por SARSCOV-2.

\section{AGRADECIMENTO}

Agradecemos a Rafaela Fiquene, por toda atenção. 


\title{
COVID-19 INFECTION DURING PREGNANCY: EVALUATION OF CLINICAL MANIFESTATIONS AND GESTATIONAL OUTCOMES
}

\begin{abstract}
The new coronavirus pandemic (SARS-COV-2) and its respiratory disease (COVID-19) soon became a global threat. As it is a new disease, a search for evidence that indicates adequate assessment and management is essential, especially in risk groups. Therefore, this work wants to elucidate and analyze the current knowledge about the impact of the viremia on pregnancy for it's proper handling. A systematic review was made, which had as a guiding principle the analysis of the clinical manifestations and complications that occurred in pregnant women infected with SARS-CoV-2. For this, the descriptors and the term MESH were used: "Pregnancy" AND "Coronavirus" through the MEDLINE and SciELO databases. From the inclusion and exclusion criteria, 15 productions were selected. The most common manifestations were similar to nonpregnant women as: fever, fatigue, dyspnea, myalgia and cough. Among divergences, aggravations were noted, such as preeclampsia. Despite these contradictions in the literature and quantitative limitations of the samples, the most reported gestational complications were fetal distress, premature rupture of membranes, premature births and even fetal death. However, findings of vertical transmission were not observed. Despite the significant increase in the performance of cesarean sections, the decisions must be individualized, a COVID-19 infection is not in isolation indicative of surgical intervention. Certainly, most of the studies come from very different cultural and demographic regions, but the knowledge and analysis of the literature are essential for understanding the disease and delineating the corresponding conducts.
\end{abstract}

KEYWORDS: Coronavirus Infections. Pregnancy Complications. Signs and Symptoms.

\section{REFERÊNCIAS}

1. World Health Organization. Coronavirus disease (COVID-19) pandemic. Available at: https://www.who.int/emergencies/diseases/ novel-coronavirus-2019. Accessed June 17, 2020.

2. Smith V, Seo D, Warty R, Payne O, Salih M, Chin $\mathrm{K} \&$ Wallace E. Maternal and neonatal outcomes associated with COVID-19 infection: A systematic review. Plos one. 2020; 15(6): 1-13.

3. Thomas B, Pallivalapila A, El Kassem W, Tarannum A, Al Hail F, Rijims M, Parappil, et al. Maternal and perinatal outcomes and pharmacological management of COVID-19 infection in pregnancy: a systematic review protocol. Syst Rev. 2020; 18(9): 1-7.
4. Della Gatta AN, Rizzo R, Pilu G, Simonazzi G.Coronavirus disease 2019 during pregnancy; a systematic review of reported cases. Am J Obstet Gynecol. 2020 Jul; 223(1): 36-41.

5. Lopes de Sousa ÁF, Carvalho HEF, Oliveira LB, Schneider G, Camargo ELS, Watanabe E, et al. Effects of COVID-19 infection during pregnancy and neonatal prognosis: what is the evidence? Int J Environ Res Public Health. 2020 Jun 11; 17(11): 1-17.

6. Segars J, Katler Q, McQueen DB, Kotlyar A, Glenn T, Knight Z, et al. Prior and novel coronaviruses, coronavírus disease 2019 (COVID-19), and human reproduction: what is known? Fertil Steril. 2020 Jun; 113(6): 1140-49. 
7. Zaigham $M$, Andersson O. Maternal and perinatal outcomes with COVID-19: a systematic review of 108 pregnancies. Acta Obstet Gynecol Scand. 2020 Jul; 99(7): 823-29.

8. Diriba K, Awulachew E, Getu E.. The effect of coronavírus infection (SARS-CoV-2, MERSCoV, and SARS-CoV) during pregnancy and the possibility of vertical maternal-fetal transmission: a systematic review and meta-analysis. Eur J Med Res. 2020; 25(1): 1-14.

9. Galvão T, Pansani T, Harrad D. Principais itens para relatar Revisões sistemáticas e Metaanálises: A recomendação PRISMA. Epidemiol. Serv. Saúde. 2015 June; 24(2): 335-42.

10. Giwa AL, Desai A, Duca A. Novel 2019 coronavírus SARS-COV-2 (COVID-19): An update overview for emergency clinicians. Emerg Med Pract 2020; 22 (05): 1-28.

11. Juan J, Gil MM, Rong Z, Zhang Y, Yang H, Poon LC. Effect of coronavirus disease 2019 (COVID-19) on maternal, perinatal and neonatal outcome: systematic review. Ultrasound Obstet Gynecol. 2020 Jul; 56(1): 15-27.

12. Liu Y, Chen H, Tang K, Guo Y. Clinical manifestations and outcome of SARS-CoV-2 infection during pregnancy. J Infect. $2020 \mathrm{Fev}$; 53(20): 1-9.
13. Di Mascio D, Khalil A, Saccone G, Rizzo G, Buca $D$, Liberati $M$, et al. Outcome of Coronavirus spectrum infections (SARS, MERS, COVID-19) during pregnancy: A systematic review and meta-analysis. Am J Obstet Gynecol MFM. 2020 May; 2(2): 1-9.

14. Capobianco G, Saderi L, Aliberti S, Mondoni M, Piana A, Dessole F, et al. COVID-19 in pregnant women: A systematic review and meta-analysis. European journal of obstetrics, gynecology, and reproductive biology, September 2020; 252: 54358.

15. Ashraf MA, Keshavarz P, HosseinpourP, Erfani A, Roshanshad A, Pourdast A, et al. Coronavirus Disease 2019 (COVID-19): A Systematic Review of Pregnancy and the Possibility of Vertical Transmission. J Reprod Infertil. 2020; 21(3): 15768.

16. Chen H, Guo J, Wang C, Lou F, Yu X, Zhang $W$, et al. Clinical characteristics and intrauterine vertical transmission potential of COVID-19 infection in nine pregnant women: a retropective review of medical records. Lancet. 2020; 395: 809-15.

17. Zhu H, Wang L, Fang C, Peng S, Zhang L, Chang $\mathrm{G}$, et. al. Clinical analysis of 10 neonates born to mothers with 2019-nCov pneumonia. Transl Pediatr. 2020; 9(1): 51-60. 